\title{
Connection in the Fresh Air: A Study on the Benefits of Participation in an Electronic Tracking Outdoor Gym Exercise Programme
}

\author{
Urban Johnson $^{1}$, Andreas Ivarsson ${ }^{1}$, James Parker ${ }^{1,2}$, Mark B. Andersen ${ }^{1}$, Ingrid Svetoft ${ }^{2}$
}

Affiliations: ${ }^{1} \mathrm{H}$ almstad University, Center of Research on Welfare, Health and Sport, Halmstad, Sweden, ${ }^{2} \mathrm{Halmstad}$ University, School of Business, Engineering and Science, Halmstad, Sweden

Correspondence: U. Johnson, Centre for Research on Welfare, Health and Sport, Halmstad University, Sweden, Box 823, S-301 18 Halmstad, Sweden. E-mail: urban.johnson@hh.se

ABSTRACT This study aimed to explore whether a six-week intervention, based on participation in outdoor exercise, including activity-tracking devices and combined with individual consulting sessions, can both increase physical activity and yield positive changes in physiological and psychological health measures. A total of six participants, with a mean age of 41.2 (range 33-50 years), completed the ten-week study and the six-week intervention. The full study consisted of a four-week control/baseline and a six-week intervention period in which each participant acted as their own controls. Continuous measures of physical activity data were collected using a wrist-worn activity sensor during the ten-week study, along with pre- and post-measures of cardiovascular fitness, upper-body strength, BMI, general health, and motivation to exercise. The intervention consisted of a resistance-training programme for an outdoor gym and three motivational interviewing sessions. Effect sizes (percentage) for changes pre- to post-training were calculated. The results, because of the small sample size, are presented as individual cases, but the group, as a whole, showed average increases from baseline (pre-) to post-measures in strength (maximum row; 15.33\%), time to exhaustion (3.58\%), number of steps per day (4\%), and autonomous motivation (12\%) and average decreases in body weight (-1.08\%), fat percentage (-7.58\%), strength (chest; $-2.5 \%)$, and stress symptoms $(-2.17 \%)$. The six-week intervention programme showed promising results regarding physical activity changes. This study contributes to the limited evidence of the impact of resistance training programmes using outdoor gyms, electronic tracker, and motivational interviewing on physical activity outcomes.

KEY WORDS physical activity, physiological health, psychological well-being, computerized exercise intervention, motivational interviewing

$@$ MJSSMontenegro

HOOKING UP IN THE AIR: OUTDOOR EXERCISE

http://mjssm.me/?sekcija=article\&artid=174

\section{Introduction}

Healthy living is associated with positive levels of several different variables, such as psychological and social well-being, physiological and metabolic health, physical capacity as well as cognitive functioning (Godfrey et al., 2013). Physical activity is one key factor to improve global public health, and evidence-based interventions to promote PA are, therefore, of central importance. Research has shown that outdoor environmental exercise interventions result in an overall increase in PA (Tester \& Baker, 2009) and access to outdoor exercise equipment has been found to help increase activity levels in people who do not usually exercise (Kelly \& Fry, 2011). Supportive urban planning (e.g., free public access to outdoor gym equipment) has the potential to facilitate PA, well-being, and health, and modern health technology can have critical potentiating effects (Pratt et al., 2012). New innovative designs using modern health technology (e.g., PA and sleep-monitoring apps for smartphones) applied to outdoor exercise might attract new users and promote sustainable health behaviours within communities (Shane, Lowe, \& Ólaighin, 2014).

Environmental factors' have the potential to influence PA, and research has highlighted the importance of promoting functional facilities designed for exercise (Kahn et al., 2002). In the literature, a number of 
potentially effective facilities have been listed (e.g., outdoor fitness equipment; Chow, 2013) and using environmental applications (e.g., outdoor fitness equipment accessible to the public) as tools to facilitate PA that may be effective because they have the potential to reach a wide variety of people. Technological advances have a similar potential to positively influence PA, and the use of activity-tracking devices has been found to increase PA motivation (Bice, Ball, \& McClaran, 2016). Two recent systematic reviews have demonstrated that the use of accelerometers (Goode et al., 2017) and smartphones (Bort-Roig, Gilson, Puig-Ribera, Contreras, \& Trost, 2014) has small and modest positive effects on PA and weight loss, respectively, among adults, but more research is needed to investigate the motivational influence of popular commercial activity monitors.

One framework frequently used to understand behavioural as well as social factors and their influences on exercise motivation is the self-determination theory (SDT; Deci \& Ryan, 2004). SDT places emphasis on the social context and its ability to facilitate or thwart optimal motivation and on the extent to which behaviours are generally self-determined or externally controlled. In SDT, it is suggested that self-determined motivation and psychological well-being will be promoted when certain basic psychological needs (autonomy, competence, relatedness) are satisfied in PA/exercise settings, and interventions nourishing these needs will not only increase self-determined motivation towards exercise programmes, but also adherence to such programmes (Ng et al., 2012; Teixeira, Carraca, Markland, Silva, \& Ryan, 2012). One approach that has been effective to support behaviour change is motivational interviewing (MI; Breckon, 2015). MI targets the three key components in self-determination theory, and this approach has been found to be effective in terms of behaviour change (Hardcastle, Taylor, Bailey, Harley, \& Hagger, 2013).

Given the current state of research into accessible outdoor exercise equipment, a systematic evaluation of the effects of participation in outdoor gym exercise programmes, supported by information technology and motivational interviewing is warranted. To our knowledge, few studies have combined physical and psychological strategies within the same intervention, using an innovative mixture of modern health technology applications, activity trackers, and motivational interviewing in an outdoor environment among a healthy but physically inactive population. This study aims to explore if a six-week intervention, based on participation in outdoor exercise sessions, using activity-tracking devices and combined with individual MI sessions, can help increase physical activity. An additional aim was to investigate if participation in the intervention was associated with changes in physiological as well as psychological health measures.

\section{Methods}

\section{Participants and inclusion criteria}

Altogether six participants, working within the Halmstad, Sweden municipality, were selected for the study $($ male $=4$; female $=2)$ with a mean age of $41.2(S D=6.5)$; men $42.5(S D=6.6)$ and women $38.5(S D=7.8)$. The selection of the participants was based on voluntary choice and with the support of the Halmstad City Council. The inclusion criteria used in the selection of the participants were: (a) having a primarily sedentary job; (b) limited exercise activity in the past year; and (c) employed within Halmstad's City Council, which is relatively close to the outdoor gym.

\section{Psychological measurements}

The General Health Questionnaire-12 (GHQ-12) (Goldberg, Gater, \& Sartorius et al., 1997) is intended to measure psychological distress/well-being and assesses three dimensions: (a) positive well-being (e.g., Have you recently been able to concentrate on whatever you're doing?), (b) anxiety (e.g., Have you recently lost much sleep due to worry?), and (c) loss of confidence (e.g., Have you recently been losing confidence in yourself?). The responses are recorded on a 4-point Likert-type scale ranging from 0 (I don't agree at all) to 3 (I totally agree) with a Cronbach alpha ranging from 0.83 to 0.93 .

Motivation was measured using the Behavioral Regulation in Exercise Questionnaire-2 (BREQ-2) (Markland, \& Tobin, 2004), which contains19 items (e.g., It's important to me to exercise regularly) measured on a fivepoint Likert scale ranging between 0 (not true for me) to 4 (very true for me). The scale measures behavioural regulations through five motivation factors: amotivation, external, introjected, identified, and intrinsic motivation and the five-factor model indicated an adequate fit for measuring exercise motivation. Based on recommendations from Sebire and colleagues (2008), we created one autonomous motivation factor (identified regulation and intrinsic regulation). This factor was used in all analyses.

\section{Physiological measurements}

Body composition measurements of body mass index (BMI) and total body fat mass were measured with an eight-polar tactile-electrode impedance meter (bioelectrical impedance analysis InBody 770, Biospace Ltd, Seoul, Korea). Bioimpedance analysis has been shown to be a valid tool, in comparison to dual-energy X-ray absorptiometry, for measuring total body-fat mass (ICC female $=0.97$, ICC male $=0.93$ ) in the general middle-aged population (Ling et al., 2011). In should be noted that bioimpedance analysis may overestimate body-fat by $8 \%$ when compared to dual-energy X-ray absorptiometry. All body composition measurements were performed early in the morning, and each participant abstained from eating and drinking coffee for at least six hours prior to the testing. The modified Bruce Treadmill Test (Noon \& Dean, 2000) (time to exhaustion) was used to measure cardiovascular fitness, and muscular strength was assessed using a one-repetition maximum strength test (Levinger et al., 2007). All strength testing was performed using commercial resistance machines (Atlantis 
Inc. Quebec, Canada). The exercises selected to assess one-repetition maximum were a chest press (PWP3010 plate-loaded vertical chest press), a shoulder press (Atlantis performance converging shoulder press PE-149), and a seated row (Atlantis performance diverging row PE-137). Due to the similarity of exercise movement and muscle groups used during the chest press and shoulder press, the change in muscular strength for the exercises was very similar; for this reason, we have chosen to only present the results from the chest press.

\section{Instrument}

Physical activity data were collected with a wrist-worn activity sensor (based on Apple Watch and iPhone) that collects information about each day's activities (steps taken). All participants were given one of these wristbands at the start of the study. Data were first stored locally on the smartphone and then downloaded from the Health Data App on the smartphones using the QS Access application. Apple Watch, iPhone 6, and the Health Data app have been reported to better validity $(\mathrm{ICC}=0.89)$ in comparison to five other commercially available devices for measuring the total amount of daily steps (Noon \& Dean, 2000); furthermore, the consistency of total steps by different devices of the Apple Watch Series 1 have been reported as very high ( $\mathrm{r}=0.99)$ (Wen, Zhang, Liu, \& Lei, 2017). A specific web-port0061l (Quick Search) was used to collect the psychometric data.

\section{Exercise intervention}

The participants took part in an intervention aimed to increase PA. Exercise behaviours were supported through individual motivational interviewing coaching sessions and a resistance-training programme designed for use in an outdoor gym. The individual motivational interviewing coaching sessions were composed of four processes (Breckon, 2015). In the engaging processes, an establishing of connection and a therapeutic relationship was established. After that, the process of focusing was developed to maintain a detailed direction in the conversation about change to support exercise behaviour. The next process was evoking, which involved the participants own motivation for change. The last process was planning, which involved both developing commitments to change and formulating an action plan. The sessions took place between Week 8 (after four weeks of preparation and four weeks of baseline and at the start of the interventions) and Week 14 (see Table 1). When the intervention started, the participants were introduced to the outdoor gym and instructed how to use it (an instructor was present at the start of the intervention for each participant). During their exercise, it was possible for the participants to access direct feedback on their physical activities at the gym (e.g., heart rate, calories burned, session duration).

\begin{tabular}{|c|c|}
\hline Week/s & Working issue \\
\hline 0 & First meeting with participants, information about the study, ethics \\
\hline 4 & Distribution of smartwatches (pre-test,) physical tests and psychological questionnaires (1) \\
\hline 4-8 & Pre-test/base-line and control period \\
\hline 8 & $\begin{array}{l}\text { Introduction to outdoor gym, 1st motivational interviewing (MI) session, 2nd psychological } \\
\text { questionnaires }\end{array}$ \\
\hline 8-14 & Interventions period \\
\hline 14 & Physical tests, second MI session and third psychological questionnaires \\
\hline
\end{tabular}

Description of the outdoor gym

The outdoor gym consists of four stationary machines and two modules for free exercises. These are row, shoulder-press, chest-press, squat (stationary machines), as well as lunge and jumping jacks (free modules). The stationary machines have a variety of resistance levels by way of a weight plate system, which allowed the participants to select the resistance they wanted. A two-metre-high post stands at the centre of the gym (see Figure 1) and acts as a timing device. Participants selected one of two training sessions, either the 20:10 s (work: rest) or the 40:20 s (work: rest) session by pressing a button on the post. Once a session was selected, LEDs inside the post glow either green or red to indicate a work period (green) or rest (red) period. The surface of the outdoor gym

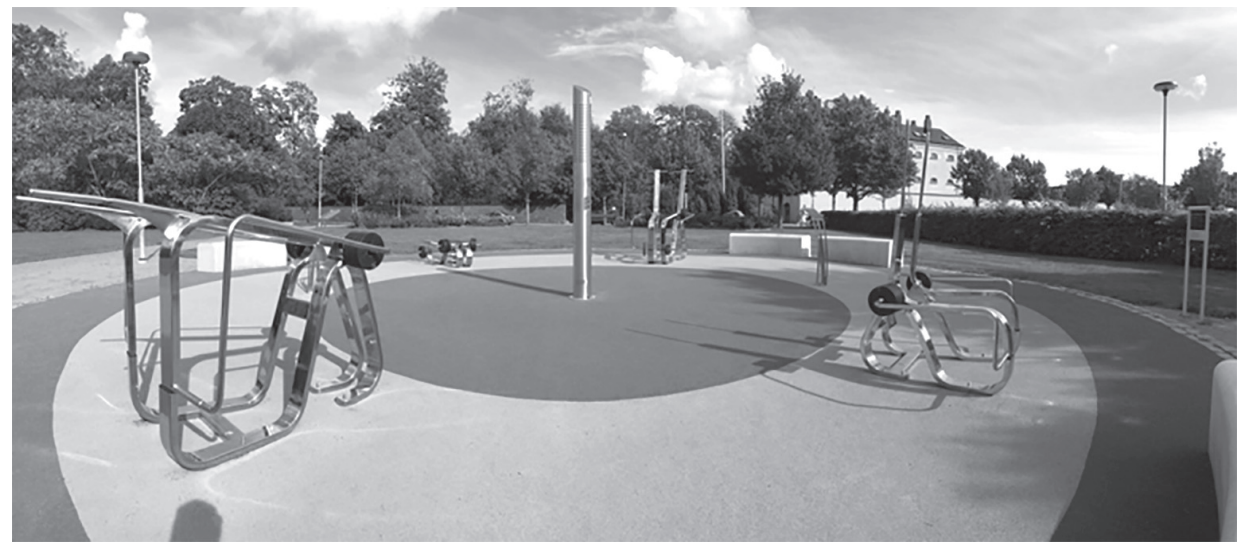

FIGURE 1 Outdoor exercise setting at Slottsparken, Halmstad, Sweden 
consists of tempered rubber in colour tones in harmony with the surrounding city park and local architecture (see Figure 1). Situated in the centre of a park with frequently used walkways, the outdoor gym is meant to invite (perhaps inspire) citizens to use it.

\section{Procedures}

Table 1 outlines the time plan for the study procedures from the first contact with the participants until the final testing session 14 weeks later. The first four weeks were dedicated to contacting participants and distributing the wrist-worn activity sensor. Baseline measures were taken during Week 5 and up to Week 8 . The intervention started in Week 8. At this point participants were instructed to complete the exercises at the outdoor gym approximately two times a week (Table 2) and, if possible, in connection with their regular work schedule (e.g., during lunchtime). Motivational interviewing occurred at Weeks 8 and 14. Ethical approval for the study was granted by the regional ethics committee (reference number 2016/843).

\begin{tabular}{lll}
\multicolumn{3}{l}{ TABLE 2 Typical training schedule for the 8-week intervention } \\
Week & Session 1 & Session $\mathbf{2}$ \\
\hline 8 & 2 rounds of $20: 10$ & 2 rounds of $20: 10$ \\
9 & 3 rounds of $40: 20$ & 3 rounds of $40: 20$ \\
10 & 4 round of $40: 20$ & 3 rounds of $40: 20$ \\
11 & 3 rounds of $40: 20$ & 4 rounds of $20: 10$ \\
12 & 4 rounds of $40: 20$ & 3 rounds of $40: 20$ \\
13 & 4 rounds of $0: 20$ & 3 rounds of $40: 20$ \\
14 & & 4 rounds of $40: 20$ \\
\hline
\end{tabular}

Note. 1 round= 1 set on each of the six stations at the outdoor gym, 20:10 $=20$ s work and 10 s rest, 40:20 $=40$ s work and 20 s rest

\section{Data analysis}

To analyse how participation in the intervention programme influenced PA motivation and exercise behaviours, we used percentages as the magnitude indices. More specifically, percentages were used to illustrate potential changes in the psychological, behavioural, and physiological measures between the baseline (pre) and Week 14 (post) measurements. These calculations were performed for each participant. The results for each variable for each participant were then summed and averaged for an overall percentage for the full sample. The results, however, are described in detail for each participant.

\section{Results}

The overall results showed an average increase from baseline to the post measures in strength (row; 15.33\%), time to exhaustion (3.58\%), steps (4\%), and autonomous motivation (12\%). The overall results also showed

TABLE 3 Cases and effect size pre-post measures (percentage)

\begin{tabular}{|c|c|c|c|c|c|c|}
\hline Participants & A & B & $\mathbf{C}$ & D & $\mathbf{E}$ & $\mathbf{F}$ \\
\hline \multicolumn{7}{|l|}{ Activity wrist-band } \\
\hline Steps (SD) Pre & $8250(3800)$ & $8440(2420)$ & $8980(2920)$ & $11100(4190)$ & $9920(5502)$ & $9190(3400)$ \\
\hline Steps (SD) Post & $8980(4050)$ & $9470(3540)$ & $9010(3940)$ & $11310(4200)$ & $10300(2770)$ & $9320(3980)$ \\
\hline Change in steps (\%) & $9 \%$ & $12 \%$ & $0.5 \%$ & $2 \%$ & $4 \%$ & $1.5 \%$ \\
\hline Difference between Avg. Steps for Age and Gender Pre & -500 & -1500 & -1000 & 1010 & 1300 & -800 \\
\hline Difference between Avg. Steps for Age and Gender Post & 230 & -470 & -970 & 1220 & 1680 & -670 \\
\hline \multicolumn{7}{|l|}{ Impedance meter } \\
\hline Body-weight (kg) Pre/Post & $89 / 89.1$ & $115 / 111$ & $93.9 / 95.2$ & $97.6 / 97.2$ & $72.4 / 72$ & $103.4 / 100.6$ \\
\hline BMI Pre/Post $\left(\mathrm{kg} / \mathrm{m}^{2}\right)$ & $28.4 / 28.4$ & $34.4 / 33.2$ & $25.7 / 26.1$ & $30.8 / 30.7$ & $25.3 / 25.2$ & $29.6 / 28.8$ \\
\hline Change body-weight (\%) & $0 \%$ & $-3.5 \%$ & $1 \%$ & $-0.5 \%$ & $-0.5 \%$ & $-3 \%$ \\
\hline Fat (kg) Pre/Post & $32 / 29.5$ & $42.8 / 38.2$ & $13.9 / 13.3$ & $27.2 / 25.7$ & $23.2 / 22.6$ & $28.3 / 24.3$ \\
\hline Change fat mass $(\%)$ & $-8 \%$ & $-11 \%$ & $-4.5 \%$ & $-5.5 \%$ & $-2.5 \%$ & $-14 \%$ \\
\hline \multicolumn{7}{|l|}{ Bruce treadmill test } \\
\hline Time to exhaustion (minutes) Pre/Post & $17: 00 / 17: 30$ & $15: 10 / 15: 38$ & $20: 39 / 20: 45$ & 16:12/18:01 & $16: 40 / 16: 48$ & $16: 27 / 17: 20$ \\
\hline Change in time to exhaustion (\%) & $2 \%$ & $2 \%$ & $0 \%$ & $11.5 \%$ & $0.5 \%$ & $5.5 \%$ \\
\hline Days without logging any activity. (Between test $1 \& 2$ ) & 0 & 0 & 6 & 1 & 0 & 0 \\
\hline \multicolumn{7}{|l|}{ Strength tests } \\
\hline Change in Chest (\%) & $0 \%$ & $10.5 \%$ & $-18 \%$ & $18 \%$ & $-8 \%$ & $-14.5 \%$ \\
\hline Change in Seated row (\%) & $13.5 \%$ & $21 \%$ & $10 \%$ & $14.5 \%$ & $27 \%$ & $7 \%$ \\
\hline \multicolumn{7}{|l|}{ Psychological questionnaire } \\
\hline Change in autonomous motivation. BREQ-2 Pre/Post (\%) & $\begin{array}{c}1.75 / 2.13 \\
(21 \%)\end{array}$ & $\begin{array}{c}2.0 / 2.83 \\
(41 \%)\end{array}$ & $\begin{array}{c}2.88 / 2.70 \\
(-6 \%)\end{array}$ & $\begin{array}{c}2.0 / 2.63 \\
(31 \%)\end{array}$ & $\begin{array}{c}2.88 / 2.55 \\
(-11 \%)\end{array}$ & $\begin{array}{c}3.0 / 2.88 \\
(-4 \%)\end{array}$ \\
\hline Change in stress symptoms, GHQ-12 Pre/Post (\%) & $\begin{array}{c}2.66 / 2.16 \\
(-19 \%)\end{array}$ & $\begin{array}{c}2.0 / 1.66 \\
(-17 \%) \\
\end{array}$ & $\begin{array}{c}1.66 / 1.16 \\
(-30 \%)\end{array}$ & $\begin{array}{c}1.33 / 1.16 \\
(-13 \%)\end{array}$ & $\begin{array}{c}2.0 / 2.16 \\
(8 \%)\end{array}$ & $\begin{array}{c}1.16 / 1.83 \\
(58 \%)\end{array}$ \\
\hline
\end{tabular}


a decrease from baseline to the post measures in body weight $(-1.08 \%)$, body fat $(-7.58 \%)$, strength (chest; $-2.5 \%)$, and stress symptoms $(-2.17 \%)$. There was a consistent direction of effects for all participants in the change of body fat, strength (row), and time to exhaustion. In all other measures, the results showed mixed directions of the effects (see Table 3 for a comprehensive overview).

\section{Discussion}

Increases in the number of steps between pre- and post- measurement together with decreases in body fat and improved physical strength in the rowing test all indicate that the six-week exercise intervention period resulted in benefits, ultimately influencing physiological and psychological well-being in positive ways. Similar results have been shown in a recent meta-analysis, evaluating the effects of short-term exercise interventions for patients with chronic heart failure (Zhang et al., 2016).

One potential explanation for the increase in PA might be the use of activity-tracking devices to reinforce autonomous motivation for outdoor activity. The use of such devices may have stimulated the participants to take part in exercise activities (Bice, Ball, \& McClaran, 2016). More specifically, applying self-monitoring to track personal improvement as well as achieving challenging goals has been suggested to be related to increased levels of autonomous motivation for PA (Kruger, Carlson, \& Kohl, 2007). Also, studies have found that the use of activity trackers can foster behaviour change via increased levels of basic psychological needs, (Nurmi, Hagger, Haukkala, Araújo-Soares, \& Hankonen, 2016). The fulfilment of these three needs is, as previously mentioned, related to increased levels of autonomous motivation.

Another potential explanation for the increased levels of PA might be that the participants took part in the individual motivational interviewing (MI) coaching sessions. Previous studies have shown that MI can strengthen a person's self-reliance for behaviour change (Hardcastle, Taylor, Bailey, Harley, \& Hagger, 2013) to increased PA. Also, in this case, the potential mechanisms for the link between MI and PA might be increased levels of the basic psychological needs as well as autonomous motivation.

Still another potential explanation for the increase in PA during the intervention period is the location of the gym. The pleasant surroundings that frame the exercise setting (a green park) and the closeness to the city centre and workplaces may have also indirectly increased motivation to exercise and helped the participants to overcome potential barriers to PA and exercise such as limited opportunities for training and time constraints.

Despite the current study's inclusion criteria, of a sedentary job and low levels of PA, our cases showed varying levels of physiological health. Regardless of participants' starting health status, the short-term exercise intervention seems to have had positive physiological and psychological outcomes. In this study, the observed effects on physiological and psychological well-being may be related to exercise decreasing psychological stress symptoms. Perhaps the participants also had a feeling that the outdoor settings were restorative (Hug, Hartig, Hansmann, Seeland, \& Hornung, 2009) and that the physical exercising represented opportunities for social interaction with other colleagues from their workplaces in a new and supportive environment. From an SDT perspective, social interaction at the outdoor gym may well have helped to support basic psychological needs such as competence and relatedness. The participants may have all selected a moderate intensity level relative to their current fitness status, and it could be that acute short-term effects of outdoor exercise have a positive influence on psychological well-being, regardless of initial fitness level. This line of reasoning receives partial support in a systematic review suggesting that public parks and green spaces have direct and positive influences on well-being (Bowler, Buyung-Ali, Knight, \& Pullin, 2010).

\section{Study limitations}

One potential study limitation, in relation to SDT, is the possibility of other mechanisms (outside of motivation) contributing to change. Nevertheless, we have based our selection of motivation as one of our key variables because this variable, in previous studies of health behaviour change, has been found to be a central component. The current study used the number of steps as a measure for PA, but this measure does not account for total PA (such as cycling, swimming, and heavy lifting) and may underestimate total PA for some participants. Another limitation that might decrease the generalizability of the results is the small sample size, which arguably makes any conclusions regarding the predictive value of the measured variables hazardous. Clearly, the findings in the current study need to be replicated in new samples.

In contrast, the practical value of the research findings is very limited if clinically significant results only can be obtained when large numbers of participants are involved. Still another limitation relates to the selection of participants. Participants' starting physical capacity varied, which allowed some participants to improve their physical strength and endurance rather quickly, whereas improvements would be less pronounced for better physically trained participants. In follow-up studies, therefore, it is likely to be necessary to have more homogeneous groups in terms of strength and fitness. In addition, because of the voluntary choice of participants, a possible dose-response pattern is conceivable in relation to previous physical activity levels. The dose-response between volume and intensity could have a relation to our results; however, a recent systematic review and meta-analysis on the dose-response of walking on cardiovascular disease factors (including aerobic fitness and BMI) concluded that there is insufficient evidence to make any conclusions on dose-response relationships (Oja et al., 2018). One of the strengths of the study is the combination of both physical and psychological measurements, allowing a multifactorial assessment of the intervention programme and the usefulness of the results. 


\section{Conclusions}

The results of the six-week intervention programme showed promising outcomes regarding PA increases in an outdoor gym setting. More specifically, most of the participants showed improved levels on both PA as well as physiological and psychological measures after the intervention. The study contributes to the limited evidence of the impact of resistance training programmes using outdoor gyms and motivational interviewing on physical activity outcomes.

\section{Funding/support}

The study was financed by a grant from The Knowledge Foundation, Sweden [Grant number 20160097].

\section{Acknowledgements}

The authors are particularly grateful to Erik Blomberg and Camilla Schough at Eleiko Sport AB, Sweden, Erik Viberg, Anton Bärwald and Pelle Wiberg at Swedish Adrenaline, Sweden, and Sofia Warpman at Halmstad Municipality, Sweden for helping to make the research project possible.

\section{REFERENCES}

Bice, M.R., Ball, J.W., \& McClaran, S. (2016). Technology and physical activity motivation. International Journal of Sport \& Exercise Psycholology, 14, 295-304. doi:10.1080/1612197X.2015.1025811

Bort-Roig, J., Gilson, N.D., Puig-Ribera, A., Contreras, R.S., \& Trost, S.G. (2014). Measuring and influencing physical activity with smartphone technology: A systematic review. Sports Medicine, 44, 671-686. doi:10.1007/s40279-014-0142-5

Bowler, D.E., Buyung-Ali, L.M., Knight, T.M., \& Pullin, A.S. (2010). A systematic review of evidence for the added benefits to health of exposure to natural environments. BMC Public Health, 10, 456. doi:10.1186/1471-2458-10-456

Breckon, J. (2015). Motivational interviewing, exercise, and nutrition counseling. In: M.B., Andersen, S.J., Hanrahan (Eds.) Doing Exercise Psychology (pp. 75-100). Champaign, IL: Human Kinetics.

Chow, H. (2016). Outdoor fitness equipment in parks: a qualitative study from older adults' perceptions. BMC Public Health,13:e1216. doi:10.1186/1471-2458-13-1216

Deci, E.L., \& Ryan, R.M. (2000). The "what" and "why" of goal pursuits: Human needs and the self-determination of behavior. Psychological Inquiry, 4, 227-268. doi:10.1207/S15327965PLI1104_01

Lara, J., Godfrey, A., Evans E, Heaven, B., Brown, J.E., Barron, E.,... Mathers, J.C. (2013). Towards measurement of the healthy ageing phenotype in lifestyle-based intervention studies. Maturitas, 76, 189-199. doi:10.1016/j.maturitas.2013.07.007

Goldberg, D.P., Gater, R., Sartorius, N., Sartorius, N., Ustun, T. B., Piccinelli, M., Gureje, O.,...Rutter, C. (1997). The validity of two versions of the GHQ in the WHO study of mental illness in general health care. Psychological Medicine, 27,191-197. doi:10.1017/S0033291796004242

Goode, A.P., Hall, K.S., Batch, B.C., Huffman, K. M., Hastings, S. N., Allen, K. D.,...Gierisch, J. M. (2017).The impact of interventions that integrate accelerometers on physical activity and weight loss: A systematic review. Annals of Behavioral Medicine, 51, 79-93. doi:10.1007/s12160-016-9829-1

Hardcastle, S.J., Taylor, A.H., Bailey, M.P., Harley, R.S., \& Hagger, M.S. (2013). Effectiveness of a motivational interviewing intervention on weight loss, physical activity and cardiovascular disease risk factors: a randomized controlled trail with a 12-month post-intervention follow-up. International Journal of Behavioral Nutrition and Physical Activity, 10, e40. doi:10.1186/1479-5868-10-40

Hug, S.M., Hartig, T., Hansmann, R., Seeland, K., \& Hornung, R. (2009). Restorative qualities of indoor and outdoor exercise settings as predictors of exercise frequency. Health \& Place, 15, 971-980. doi:10.1016/j. healthplace.2009.03.002

Kahn, E.B., Ramsey, L.T., Brownson R.C., Heath, G.W., Howze, E.H., Powell, K.E.,...Corso, P. (2002). The effectiveness of interventions to increase physical activity. American Journal of Preventive Medicine, 22, 73-107. doi.org/10.1016/S0749-3797(02)00434-8

Kelly, B., \& Fry, J. (2011). Camden outdoor gyms evaluation: Phase 1 [pdf]. London: NHS Camden. Available from http://www.camdendata.info/

Kruger, J., Carlson, S.A., \& Kohl, H.W. (2007). Fitness facilities for adults: Differences in perceived access and usage. American Journal of Preventive Medicine, 32, 500-505. doi:10.1016/j.amepre.2007.02.003

Lara, J., Godfrey A., Evans E., Heaven, B., Brown, L. J., Barron, E.,... Mathers, J. C. (2013). Towards measurement of the healthy ageing phenotype in lifestyle-based intervention studies. Maturitas, 7(6), 189-199. doi:10.1016/j.maturitas.2013.07.007

Levinger, I., Goodman, C., Hare, D., Jerums, G., Toi, D., \& Selig, S. (2007). The reliability of the 1RM test for untrained middle-aged individuals. Journal of Science and Medicine in Sport, 12, 310-316. doi:10.1016/j. jsams.2007.10.007

Ling, C.H.Y., de Craen, A. J.M., Slagboom, P.E., Gunn, D.A., Stokkel, M.P.M.,Westendorp, R.G.J.,...Maier, A.B. (2011). Accuracy of direct segmental multi-frequency bioimpedance analysis in the assessment of total body and segmental body composition in middle-aged adult population. Clinical Nutrition, 30, 610615. doi:10.1016/j.clnu.2011.04.001

Markland, D., \& Tobin, V. (2004). A modification to the Behavioral Regulation in Exercise Questionnaire to 
include an assessment of amotivation. Journal of Sport \& Exercise Psychology, 26, 191-196. doi:10.1123/ jsep.26.2.191

Ng, J.Y.Y., Ntoumanis, N., Thogersen-Ntoumani, C., Deci, E.L., Ryan, R.M., Duda, J.L., \& Williams, G.C. (2012). Self-determination theory applied to health contexts: A meta-analysis. Perspectives on Psychological Science, 7, 325-340. doi:19.1177/175691612447309

Noon, V., \& Dean, E. (2008). Submaximal exercise testing: Clinical application and interpretation. Physical Therapy, 80, 782-807. doi:10.1093/ptj/80.8.782

Nurmi, J., Hagger, M.S., Haukkala, A., Araújo-Soares, V., Hankonen, N. (2016). Relations between autonomous motivation and leisure-time physical activity participation: The mediating role of self-regulation techniques. Journal of Sport \& Exercise Psychology, 38, 128-137. doi:10.1123/jsep.2015-0222

Oja, P., Kelly, P., Murtagh, E., Murphy, M., Foster, C., \& Titze, S. (2018). Effects of Frequency, intensity, duration and volume of walking interventions on CVD risk factors: a systematic review and meta-regression analysis of randomized controlled trials among inactive healthy adults. British Journal of Sports Medicine, 52, 769-755. doi:10.1136/bjsports-2017-098558

Pratt, M., Sarmiento, O.L., Montes, F., Ogilvie, D., Marcus, B.H., Perez, L.G.,...Brownson, R.C. (2012). The implications of megatrends in information and communication technology and transportation for changes in global physical activity. Lancet Physical Activity Series Working Group. Lancet, 380, 282-293. doi:10.1016/S0140-6736(12)60736-3

Tester, J., \& Baker, R. (2009). Making the playfields even: Evaluating the impact of an environmental intervention on park use and physical activity. Prevention Medicine, 48:316-320. doi:101016/j.ypmed.2009.01.010

Shane, A., Lowe, S.H., \& Ólaighin, G. (2014). Monitoring human health behaviour in one's living environment: A technological review. Medical Engineering \& Physics, 36, 147-168. doi:10.1016/j.medengphy.2013.11.010

Sebire, S.J., Standage, M., \& Vansteenkiste, M. (2008). Development and validation of the goal content for exercise questionnaire. Journal of Sport \& Exercise Psychology, 30(4), 353-77. doi:10.1123/jsep.30.4.353

Teixeira, P.J., Carraca, E.V., Markland, D., Silva, M.N., \& Ryan, R.M. (2012). Exercise, physical activity, and self-determination theory: a systematic review. International Journal of Behavioral Nutrition and Physical Activity, 9, 78. doi:10.1186/1479-5868-9-78

Wen, D., Zhang, X., Liu, X., \& Lei, J. (2017). Evaluating the consistency of current mainstream wearable devices in health monitoring: A comparison under free-living conditions. Journal of Medicine and Internet Research, 19(3), e68. doi:10.2196/jmir.6874

Zhang, Y., Lisheng, X., Yao, Y., Sun, Y., Zhang, J., \& Fu, Q. (2016). Effects of short-term intervention on cardiovascular functions and quality of life of chronic heart failure patients: A meta-analysis. Journal of Exercise Science \& Fitness, 14, 67-75. doi:10.1016/j.jesf.2016.08.00 